\title{
Self-consistent study of the reflection component in 4U1705-44 with XMM-Newton, BeppoSAX and RXTE in the hard and soft state
}

\author{
Elise Egron** \\ Università di Cagliari - Italy \\ E-mail: elise.egron@dsf.unica.it \\ Tiziana Di Salvo \\ Università di Palermo - Italy \\ E-mail: disalvo@fisica.unipa.it

\section{Sara Motta} \\ INAF-Osservatorio Astronomico di Brera - Italy \\ E-mail: sara.motta@brera.inaf.it

\section{Luciano Burderi} \\ Università di Cagliari - Italy \\ E-mail: burderi@dsf.unica.it
}

\begin{abstract}
We perform a self-consistent study of the reflection component in the bright atoll source 4U 170544 using XMM-Newton, BeppoSAX and RXTE data. These data are not simultaneous, nevertheless the spectral properties of the source are very similar at similar flux levels. We therefore select observations performed at similar flux levels in order to study the evolution of the spectral shape from the soft to the hard state, in a broad energy band (0.1-200 keV), using self-consistent reflection models. These models provide a good fit of the X-ray spectrum in both states, in the whole spectral range. We discuss the differences in the main spectral parameters when the source goes from the hard to the soft state, providing evidence that the inner accretion disk is truncated further from the compact object in the hard state. We also study carefully the pile-up effects in the XMM-Newton data when the source was in the soft state.
\end{abstract}

The Extreme and Variable High Energy Sky

September 19-23, 2011

Chia Laguna (Cagliari), Italy

\footnotetext{
*Speaker.

${ }^{\dagger}$ This work was supported by the Initial Training Network ITN 215212: Black Hole Universe funded by the European Community.
} 


\section{Introduction}

$4 \mathrm{U} 1705-44$ is a low mass X-ray binary system (LMXB) containing a neutron star (NS) weakly magnetized, located at $7.4 \mathrm{kpc}$ toward the Galactic ridge (8), (9). In such system the accretion disk can arrive very close to the NS surface. This implies a similar configuration with respect to accreting stellar-mass black hole (BH), since the radius of the NS is close to the size of the last stable orbit of material around BH. Similarities have been observed between these systems, suggesting common physical processes producing X-ray emission and so similar properties of the accretion flow around the compact object.

A broad iron line is often detected in the X-ray spectra of LMXBs containing either a BH or a NS. However the reason of the large width of the line is still debated (12),(2). It can be produced by reflection in the accretion disk (16), (11), or it can be ascribed to a Compton broadening in the hot corona (1).

$4 \mathrm{U}$ 1705-44 has been deeply studied with the particular objective to understand the origin and the nature of the iron line. A broad iron line was clearly detected with Chandra (5), then confirmed with BeppoSAX (7), (13). In addition to this, a signature of Compton reflection: a reflection hump peaking at nearly $30 \mathrm{keV}$ has been detected with BeppoSAX and INTEGRAL (7). Due to their large effective areas and good energy resolution, Suzaku and XMM-Newton observed a skewed Fe K $\alpha$ emission line in $4 \mathrm{U} 1705-44$ (14), (4). The spectrum obtained with XMM-Newton is consistent with a disk-reflection scenario, in agreement with the broadening of the iron line explained by Doppler and relativistic effects in the inner parts of the accretion disk. Also the presence of several emission lines at $2.62 \mathrm{keV}, 3.31 \mathrm{keV}$ and $3.90 \mathrm{keV}$ identified as S XVI, Ar XVIII and Ca XIX, respectively, and an absorption edge at $8.5 \mathrm{keV}$ are consistent with the reflection scenario (4), (3).

Here we present the spectral analysis of the bright and atoll source 4U 1705-44 using the data of XMM-Newton, BeppoSAX, and RXTE when the source was in the hard state and in the soft state. Using a same model in both states, we can follow the evolution of the spectral parameters and see that the accretion disk is truncated further from the compact object in the hard state.

\section{Observations}

The light curve produced with the All-Sky Monitor on-board RXTE allows to follow the evolution of the source on about 16 years. The source shows clear spectral transitions, from the hard ( 3 counts/s) to the soft state ( 25 counts/s). Four observations were performed with BeppoSAX and $X M M-N e w t o n$ when the source was in the soft and hard state (Fig.1). The spectral transitions are associated with variations in the X-ray flux. We select RXTE observations, both in the hard and soft state, as shown in Fig. 1. In this way, using three different satellites, we cover a broad-band energy from 0.1 to $200 \mathrm{keV}$.

\section{Spectral analysis}

\subsection{Soft State}

We re-analyzed separately the XMM/EPIC-pn data of 4U 1705-44 during the soft state in order to assess whether the iron line is a relativistic line. We study carefully the pile-up effects 

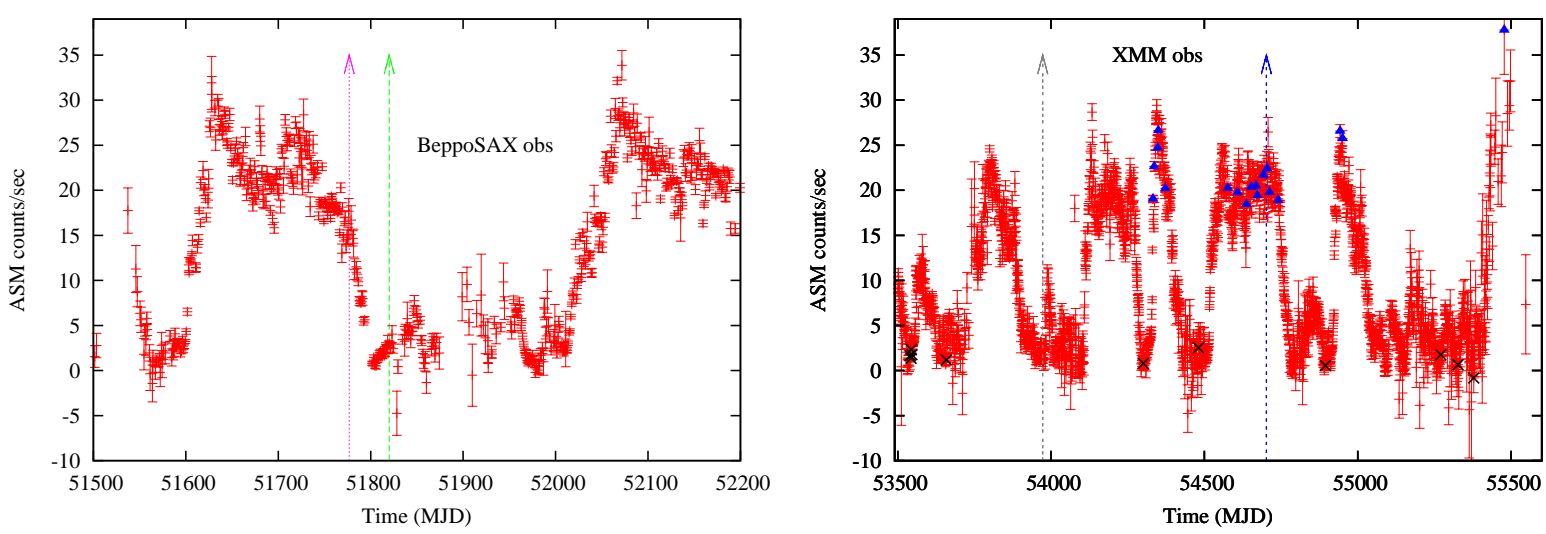

Figure 1: RXTE/ASM light curves of 4U 1705-44 from November 1999 to October 2001, and from May 2005 to November 2010. Left panel: The two arrows show the observations performed by BeppoSAX in August and October 2000. Right panel: The arrows mark the two XMM-Newton observations performed in August 2006 and 2008. The black crosses and the blue triangles represent the RXTE observations selected in the hard and soft state, respectively.

in this observation. Performing a comparison between the pn spectra excluding 1, then 2, until 7 central columns of the CCD, we conclude that the effects of pile-up are removed when we delete 2 central columns. Moreover, the iron line does not seem to be affected by pile-up. Details about this analysis are given in Egron et al. (in prep). In the following analysis, we use the EPIC/pn data where 2 central columns have been excluded.

Considering separately the data of each satellite, the continuum of the XMM-Newton and BeppoSAX spectra is well fitted by a blackbody plus a thermal Comptonized model (compTT or NTHсомP) modified at low energy by the interstellar photoelectric absorption (PHABs). In both cases, the fit is improved by adding a broad iron line, using a Gaussian line or even better a diskline at 6.7 $\mathrm{keV}$. In the case of the XMM-Newton data, other broad emission lines are detected at lower energy, at 2.60, 3.31 and $3.90 \mathrm{keV}$, associated to S XVI, Ar XVIII and Ca XIX, respectively. These lines are well fitted by gaussians or disklines, which have the same smearing parameters than the iron line (the inclination, the emissivity index and the inner and outer radii of the disk). In addition to this, an iron edge is also present at about $8.5 \mathrm{keV}$. The $\chi_{\text {red }}^{2}$ obtained with XMM-Newton and BeppoSAX data using disklines are 1.03 (409 dof) and 1.16 (503 dof) respectively, which are better than using a simple gaussian to fit the iron line (1.14 (408 dof) and 1.23 (505 dof) respectively). The F-test associated to the BeppoSAX fits gives a probability of chance improvement of about $10^{-7}$. The values of the parameters are similar to each other and they are in agreement with the previous results reported by (4) and (13). The inclination of the system estimated with the XMM-Newton data is $i=39_{-1}^{+2 \circ}$ and with BeppoSAX, $i=28_{-5}^{+8 \circ}$. The inner radii of the accretion disk associated are $R_{\mathrm{in}}=$ $14_{-2}^{+3} R_{\mathrm{g}}$ and $R_{\mathrm{in}}=8_{-2}^{+4} R_{\mathrm{g}}$, respectively. Note that the uncertainties on the inner radius and on the inclination are lower in the case of XMM-Newton. This can be explained by the quality of the data which is better in the case of XMM-Newton due to its higher energy resolution and larger effective area.

Since the values of the parameters are very similar between XMM-Newton and BeppoSAX, we 
fitted these data simultaneously, adding also the RXTE data. The different cross calibrations of the different instruments were taken into acccount by including normalizing factors in the model. These factors were fixed to 1 for $\mathrm{pn}$ and kept free for the other instruments.

In order to include all the reflection features we use a self-consistent reflection model, Reflionx (15). However this model does not include Ar XVIII and Ca XIX, neither the edge at $8.5 \mathrm{keV}$, that is why we add two gaussians and an edge to take into account these features. We multiply reflionx with a high energy cutoff, where the cutoff energy is frozen to $0.1 \mathrm{keV}$ and the fold energy is 2.7 times the temperature of the electrons. To take into account the smearing of the iron line induced by Doppler and by the relativistic effects close to the compact object, we convolve the reflection model, the two gaussians and the edge with the same rdblur component (6).

We let some parameters free to vary from one instrument with respect to the others, such as the column density, the parameters of nthComp (the photon index, the temperature of the electrons, of the seed photons, and the normalization) and the fold energy which are slightly different from BeppoSAX and XMM-Newton. In the case of BeppoSAX, $n_{\mathrm{H}}=1.9 \times 10^{22} \mathrm{~cm}^{-2}, \Gamma=2.24, T_{\mathrm{e}}=2.87$ $\mathrm{keV}, T_{\mathrm{bb}}=1.11 \mathrm{keV}$, that we can compare to those given by $X M M$-Newton, $n_{\mathrm{H}}=2.03 \times 10^{22} \mathrm{~cm}^{-2}$, $\Gamma=2.55, T_{\mathrm{e}}=2.93 \mathrm{keV}, T_{\mathrm{bb}}=1.28 \mathrm{keV}$. As regards $R X T E$ only the column density and the photon index are free to vary $\left(n_{\mathrm{H}}=3.7 \times 10^{22} \mathrm{~cm}^{-2}\right.$ and $\left.\Gamma=2.43\right)$. The $\chi^{2} /$ dof obtained is $1838 / 1573$ $(\sim 1.17)$. The values of the parameters associated to this model are reported in Table 1 .

\subsection{Hard State}

In this section we apply the same procedure used for the soft state to $4 \mathrm{U} 1705-44$ data when the source was in the hard state using the three satellites: XMM-Newton, BeppoSAX and RXTE.

Regarding the $X M M$-Newton data, we excluded a type-I X-ray burst before performing the spectral analysis. The study of the burst is described by (3).

We use the same continuum model than in the soft state on the data in the hard state in order to see the evolution of the parameters. The temperature of the electrons is about $14-16 \mathrm{keV}$, the temperature of the seed photons is $1.0-1.2 \mathrm{keV}$, the optical depth of the Comptonized component is $5-6$ and the blackbody is found at $0.55-0.58 \mathrm{keV}$. The addition of a gaussian improves considerably the fit. A broad iron line is present in all the data at nearly $6.4-6.6 \mathrm{keV}$. While for the $X M M$-Newton data, the $\chi^{2} /$ dof is $405 / 419(\sim 0.97)$ using a gaussian, there are still some residuals at higher energy in the case of BeppoSAX and RXTE. The associated $\chi_{\text {red }}^{2}$ are 1.24 (484 dof) and 1.15 (65 dof) respectively. Using a diskline instead of a gaussian we have to freeze the values of the outer radius to $3500 R_{\mathrm{g}}$ and the inclination of the system to $39^{\circ}$, as in the soft state and in agreement with (4). The $\chi_{\text {red }}^{2}$ are the same (0.97 (418 dof), 1.24 (483 dof) and 1.15 (65 dof) for XMM-Newton, BeppoSAX and RXTE respectively).

We apply a self-consistent reflection model on the data (Refionx) in order to compare the values of the parameters in the hard state and in the soft state, including normalizing factors to take into account the different cross calibrations of the different instruments. We convolve this model with the rdblur component to include relativistic effects present in the inner disk. Since we only detect the iron line, we did not add any other emission line or edge. We let some parameters free to vary between the instruments, such as the column density, the blackbody temperature which is $k T_{\mathrm{bb}}=0.25 \mathrm{keV}$ for all the instruments excepted for XMM-Newton where $k T_{\mathrm{bb}}=0.57 \mathrm{keV}$. Also, in the case of RXTE, we had to let the photon index of the power-law and the electron temperature 
free to obtain such a good fit. The photon index corresponding is 2.10 whereas in the other cases $\Gamma=1.85$, and the electron temperature is found higher $\left(k T_{\mathrm{e}}=75 \pm 30 \mathrm{keV}\right.$ instead of $k T_{\mathrm{e}}=23 \pm 2$ $\mathrm{keV}$ for the other instruments).

The inclination angle is not well constrained so we freeze its value to $39^{\circ}$, value obtained for the soft state. Also the outer radius of the accretion disk is frozen at $3500 R_{\mathrm{g}}$. This value corresponds to the best estimate obtained by (4). We freeze the fold energy of the high energy cutoff to the electron temperature of the nthcomp component.

We obtain a good fit and interesting values of the parameters such as the inner radius of the accretion disk, which is truncated quite far from the neutron star surface $\left(R_{\text {in }}=26-65 R_{\mathrm{g}}\right.$ with Reflionx), the high temperature of the electrons (about $23 \mathrm{keV}$ ), and a low value of the ionization parameter $\left(\xi \sim 200 \mathrm{erg} \mathrm{cm} \mathrm{s}^{-1}\right)$.
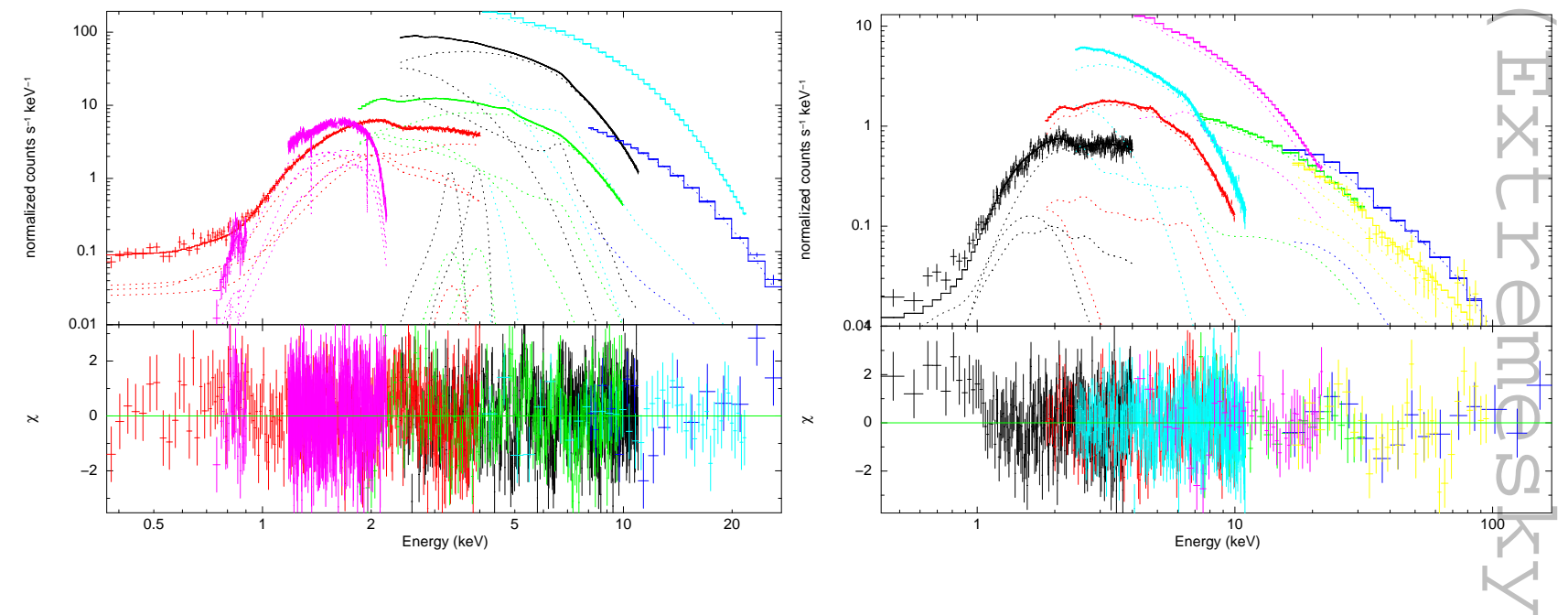

Figure 2: Spectra of 4U 1705-44 obtained with XMM-Newton, BeppoSAX, and RXTE in the soft and hard state. The bottom panels show the residuals (data-model) in unit of $\sigma$ when the reflionx model is applied. The parameters associated to these spectra are reported in Table 1. Left panel: XMM/EPICpn (black), BeppoSAX/LECS (red), BeppoSAX/MECS (green), BeppoSAX/HPGSPC (blue), RXTE/PCA (cyan), XMM/RGS1 (magenta) data points in the range 0.3-30 keV, when 4U 1705-44 was in the soft state. Right panel: BeppoSAX/LECS (black), BeppoSAX/MECS (red), BeppoSAX/HPGSPC (green), BeppoSAX/PDS (blue), XMM/EPIC-pn (cyan), RXTE/PCA (magenta), RXTE/HXTE (yellow) data points in the range $0.4-200 \mathrm{keV}$, corresponding to the hard state.

\section{Discussion}

We performed the spectral analysis of 4U 1705-44 from XMM-Newton, BeppoSAX and RXTE data, both in the hard state and in the soft state. We are able to fit these data from different instruments, which are not simultaneous, with self-consistent reflection models. Due to the good spectral coverage from the low $(0.1 \mathrm{keV})$ to the high energy $(200 \mathrm{keV})$ we have good constraints on the continuum emission and on the reflection features, allowing to follow the evolution of the spectral parameters from the hard state to the soft state. 


\subsection{The iron line}

The origin of the iron line in neutron star LMXBs is still debated. Based on the analysis of a sample of NS LMXBs observed by XMM-Newton, (12) conclude there is no evidence for an asymmetric line in these systems. On the other hand, (2) conclude the iron line is relativistic using XMM-Newton and Suzaku data.

We studied carefully the pile-up effects in the XMM/EPIC-pn spectrum of 4U 1705-44 during the soft state. We demonstrated that the exclusion of the 2 central columns of the CCD in the XMM-Newton data of $4 \mathrm{U} 1705-44$ is enough to get rid of pile-up effects. We also conclude that the iron line is a broad and relativistic line, in agreement with (4) and (3). Details about this analysis are given in Egron et al. (in prep).

\subsection{From the Hard State to the Soft State}

We apply self-consistent reflection models on 4U 1705-44 data obtained with XMM-Newton, BeppoSAX, and RXTE, on a broad-band range (from 0.1 to $200 \mathrm{keV}$ ). These models are calculated for an optically-thick atmosphere (such as the surface of an accretion disk) of constant density illuminated by a power-law spectrum.

In all the models, the rdblur component was necessary to improve the fit. The relativistic blurring was applied on the entire reflection spectrum, confirming the common origin of the reflection features in the inner part of the accretion disk, where strong relativistic effects can broaden emission and absorption features. This component gave us informations on the inclination of the system $i=39^{\circ}$ and on the inner radius of the accretion disk, $R_{\mathrm{in}}=11-17 R_{\mathrm{g}}$ in the soft state and $R_{\text {in }}=23-65 R_{\mathrm{g}}$ in the hard state. So we have here a clear indication that the accretion disk is close to the neutron star surface in the soft state and truncated further from the compact object in the hard state.

The spectral state transitions are also associated with variations in the X-ray luminosity. We calculated the accretion rate in both states using the typical value of the accretion efficiency $\eta=$ 0.2 corresponding to a neutron star $\left(M_{\mathrm{NS}}=1.4 M_{\mathrm{sol}}\right.$ and $\left.R_{\mathrm{NS}}=10 \mathrm{~km}\right)$ and to the bolometric luminosities deduced by the reflection models $\left(L_{\mathrm{SS}} \sim 1.8 \times 10^{38} \mathrm{erg} \mathrm{s}^{-1}\right.$ assuming a distance to the source of $7.4 \mathrm{kpc}$ and $L_{\mathrm{HS}} \sim 2 \times 10^{37} d_{7.4}^{2} \mathrm{erg} \mathrm{s}^{-1}$ ). In the soft state, $\dot{M}_{\mathrm{SS}}=1.6 \times 10^{-8} M_{\text {sol }} y r^{-1}$, and in the hard state, the accretion rate becomes lower, $\dot{M}_{\mathrm{HS}}=2 \times 10^{-9} M_{\mathrm{sol}} y r^{-1}$. This difference in the accretion rate is consistent with changes in the geometry flow and hence with a different inner radius of the accretion disk in the two states.

If we compare the Comptonization parameters, during the soft state the electron temperature is $T_{\mathrm{e}}=3-4 \mathrm{keV}$, the seed photons temperature is $T_{\mathrm{bb}}=1.0-1.3 \mathrm{keV}$, the photon index for the illuminating power-law spectrum is $\Gamma=2.2-2.8$, and during the hard state, $T_{\mathrm{e}}=23 \mathrm{keV}, T_{\mathrm{bb}}=$ $0.7-0.8 \mathrm{keV}$ and $\Gamma=1.85$. So we can clearly see the evolution of the spectral parameters from the soft to the hard state. The electron temperature increases whereas the optical depth and the temperature of the seed photons decrease. The seed photons are expected to come from the neutron star surface or from the boundary layer. This is in agreement with the hypothesis that in the hard state the hot corona is more important and the electron temperature is higher than in the soft state.

Moreover, the Reflionx model gave us an indication of the ionization state of the matter in the inner part of the accretion disk : $\xi=4 \pi F_{\mathrm{X}} / n_{\mathrm{H}}$, where $F_{\mathrm{X}}$ is the total illuminating flux $\left(\mathrm{erg} \mathrm{cm}^{-2} \mathrm{~s}^{-1}\right.$ ) 
and $n_{\mathrm{H}}$ is the hydrogen number density. $\xi=2700 \mathrm{erg} \mathrm{cm} \mathrm{s}^{-1}$ in the soft state and $\xi=210 \mathrm{erg} \mathrm{cm}$ $\mathrm{s}^{-1}$ in the hard state, indicating the matter is much more ionized in the soft state. This is consistent with the iron line found in the soft state: $E_{\mathrm{Fe}}=6.6-6.7 \mathrm{keV}$ and in the hard state $E_{\mathrm{Fe}}=6.4-6.5$ $\mathrm{keV}$.

\section{References}

[1] Asai K., Dotani T., Nagase F. et al. 2000, ApJS, 131, 571

[2] Cackett E. M., Miller J. M., Ballantyne D. R. et al. 2010, ApJ, 720, 205

[3] D’Ai A., Di Salvo T., Ballantyne D. et al. 2010, A\&A, 516, 36

[4] Di Salvo T., D’Ai A., Iaria R. et al. 2009, MNRAS, 398, 2022

[5] Di Salvo T., Iaria R., Mendez M. et al. 2005, ApJ, 623, L121

[6] Fabian A. C., Rees M. J., Stella L. et al. 1989, MNRAS, 238, 729

[7] Fiocchi M., Bazzano A., Ubertini P. et al. 2007, ApJ, 657, 448

[8] Forman W., Jones C., Cominsky L. et al. 1978, ApJS, 38, 357

[9] Galloway D. K., Muno M. P., Hartman J. M. et al. 2008, ApJS, 179, 360

[10] Garcia J., Kallman T. R. 2010, ApJ, 718, 695

[11] Matt G. 2006, AN, 327, 949

[12] Ng C., Diaz Trigo M., Cadolle Bel M., et al. 2010, A\&A, 522, 96

[13] Piraino S., Santangelo A., Di Salvo T. et al. 2007, A\&A, 471, L17

[14] Reis R. C., Fabian A. C., Young A. J. 2009, MNRAS, 399, L1

[15] Ross R. R. \& Fabian A. C. 2005, MNRAS, 358, 211

[16] Reynolds C. S., Nowak M. A. 2003, Physics Reports, 377, 389 
Table 1: Comparison of the self-consistent, relativistically smeared reflection model reflionx applied on the 4U 1705-44 data of XMM-Newton, BeppoSAX and RXTE in the soft state and in the hard state. The model is phabs*rdblur*edge*(bbody+nthcomp+gauss+gauss+highecut*reflionx).

\begin{tabular}{|c|c|c|c|}
\hline Component & Parameter & Soft state & Hard state \\
\hline phabs & $N_{\mathrm{H}}\left(\times 10^{22} \mathrm{~cm}^{-2}\right)$ & $2.06 \pm 0.01$ & $1.9 \pm 0.1$ \\
\hline rdblur & Betor & $-2.1 \pm 0.1$ & -3 (frozen) \\
\hline rdblur & $R_{\text {in }}\left(\mathrm{GM} / c^{2}\right)$ & $13 \pm 3$ & $40_{-14}^{+25}$ \\
\hline rdblur & $R_{\text {out }}\left(\mathrm{GM} / c^{2}\right)$ & 3500 (frozen) & 3500 (frozen) \\
\hline rdblur & $\mathrm{i}\left({ }^{\circ}\right)$ & $36_{-3}^{+2}$ & 39 (frozen) \\
\hline edge & $\mathrm{E}(\mathrm{keV})$ & $8.7 \pm 0.1$ & - \\
\hline edge & $\operatorname{Max} \tau\left(\times 10^{-2}\right)$ & $4.1 \pm 0.6$ & - \\
\hline bbody & $k T_{\mathrm{bb}}(\mathrm{keV})$ & $0.57 \pm 0.01$ & $0.25 \pm 0.02$ \\
\hline bbody & Norm $\left(\times 10^{-2}\right)$ & $2.6 \pm 0.01$ & $1.99 \pm 0.02$ \\
\hline nthComp & $\Gamma$ & $2.6 \pm 0.1$ & $1.85_{-0.01}^{+0.02}$ \\
\hline nthComp & $k T_{\mathrm{e}}(\mathrm{keV})$ & $3.0 \pm 0.1$ & $23_{-1.3}^{+1.7}$ \\
\hline nthComp & $k T_{\mathrm{bb}}(\mathrm{keV})$ & $1.30 \pm 0.02$ & $0.7_{-0.01}^{+0.02}$ \\
\hline nthComp & Norm & $0.14 \pm 0.01$ & $4.0 \pm 0.2$ \\
\hline gauss & $\mathrm{E}(\mathrm{keV})$ & $3.30_{-0.02}^{+0.03}$ & - \\
\hline gauss & Norm $\left(\times 10^{-3}\right)$ & $1.4 \pm 0.2$ & - \\
\hline gauss & $\mathrm{E}(\mathrm{keV})$ & $3.92 \pm 0.02$ & - \\
\hline gauss & Norm $\left(\times 10^{-3}\right)$ & $1.4 \pm 0.2$ & - \\
\hline highecut & cutof $f_{E}(k e V)$ & 0.1 (frozen) & 0.1 (frozen) \\
\hline highecut & $\operatorname{fold}_{E}(\mathrm{keV})$ & $8.9\left(=3^{*} k T_{\mathrm{e}}\right.$ of nthComp $)$ & $23\left(=k T_{\mathrm{e}}\right.$ of nthComp $)$ \\
\hline reflionx & Fe/Solar & $2.1_{-0.2}^{+0.5}$ & $1.1 \pm 0.3$ \\
\hline reflionx & $\Gamma$ & 2.6 (= $\Gamma$ of nthComp) & 1.85 (= $\Gamma$ of nthComp) \\
\hline reflionx & $\xi$ & $3370_{-860}^{+2266}$ & $209 \pm 6$ \\
\hline \multirow[t]{2}{*}{ reflionx } & Norm $\left(\times 10^{-6}\right)$ & $8_{-1}^{+3}$ & $1.6_{-0.2}^{+0.4}$ \\
\hline & Total $\chi^{2}$ red (d.o.f.) & $1.17(1573)$ & $1.09(980)$ \\
\hline
\end{tabular}

